\title{
EMBRYONIC DEVELOPMENT AND MALFORMATIONS OF LYMPHATIC VESSELS
}

\author{
J. Rössler \\ Pediatric Hematology/Oncology, Center of Paediatrics and Adolescent Medicine, Freiburg im Breisgau, \\ Germany
}

The growth of lymphatic vessels, lymphangiogenesis, has received considerable attention in the last years, due to the identification of proteins specifically expressed on lymphatic vessels and the discovery of molecules that can drive lymphatic vessel growth. Disturbance of lymphangiogenesis can lead to inborn defects of the lymphatic system.

Absence of lymphatic vessels in the extremities can cause accumulation of lymphatic liquid in the soft tissue resulting in lymphedema. Developmental defects of lymphatic vessels can lead to micro or macro cysts such as "cystic hygroma" and lymphangioma. Diffuse distribution of lymphatic vessels in several organs is defined as lymphangiomatosis. In the thorax, lymphangiomatosis can result in pleural effusions composed of chylus. Causal therapeutic options for lymphatic vessel malformations do not exist.

We investigated the origin of lymphatic endothelial cells (LECs) in human fetuses and compared the molecular profile of LECs from normal and malformed lymphatic vessels of children. In 19 week-old human fetuses, Prox1 and vascular endothelial growth factor receptor-3 (VEGFR-3) are co-expressed in LECs of lymphatic trunks and lymphatic capillaries as shown by immunofluorescence. Microarray analyses of LECs from lymphangiomas of children show a large number of regulated genes, such as VEGFR-3. Immunohistochemistry of thoracic lymphangiomatosis showed expression of VEGFR-1 and -2 .

Studies of the molecular background of defects in embryonic lymphatic vessel development can identify possible new therapeutic targets. New drugs are now available that would possibly show efficacy in lymphatic vessel malformations. 